\title{
Requirements Engineering zur Umsetzung des OZG: Status quo, Fallstudie und Optimierungspotenziale zur Realisierung kommunalrelevanter OZG-Lösungen
}

\author{
Frank Hogrebe $\mathbb{D}$
}

Eingegangen: 29. März 2021 / Angenommen: 17. Juli 2021 / Online publiziert: 13. August 2021

(C) Der/die Autor(en) 2021

Zusammenfassung Das Gesetz zur Verbesserung des Onlinezugangs zu Verwaltungsleistungen (kurz: Onlinezugangsgesetz - OZG) aus dem Jahr 2017 fordert, dass öffentliche Verwaltungen ihre Verwaltungsleistungen bis Ende 2022 auch elektronisch zugänglich machen. Zur Umsetzung dieser gesetzlichen Forderung bilden adressatenspezifische Anforderungen wesentliche lösungsrelevante Grundlagen. Bund, Länder und Kommunen haben sich zur Umsetzung des OZG auf unterschiedliche Wege gemacht, wobei den Bundesländern mit ihren Kommunen, aufgrund des Umfangs an Verwaltungsleistungen hier eine Schlüsselrolle in Deutschland zukommt. Der Beitrag arbeitet heraus, welcher Rahmen für die Bundesländer bei der OZG-Umsetzung gilt und inwiefern adressatenspezifische Anforderungen dabei zu berücksichtigen sind. Auf Basis von Vergleichsergebnissen eines großen kommunalen IT-Zweckverbandes wird gezeigt, welche Optimierungspotenziale gehoben werden können, sofern adressatenspezifische Anforderungen bereits bei der Umsetzungskonzeption einbezogen werden. Abschließend werden Handlungsempfehlungen aufgezeigt, wie gewählte Umsetzungswege in den Bundesländern mit ihren Kommunen optimiert werden könnten. Der Beitrag adressiert sowohl Führungskräfte und OZG-Leitungskräfte in der Verwaltungspraxis als auch die wissenschaftliche Community in diesem hoch dynamischen Forschungsfeld.

Schlüsselwörter Anforderungsmanagement · Fallstudie · Öffentliche Verwaltung · Onlinezugangsgesetz $\cdot$ Umsetzungswege

Frank Hogrebe $(\bowtie)$

Digitalisierung und Prozessmanagement, Hessische Hochschule für Polizei und Verwaltung,

Wiesbaden, Deutschland

E-Mail: Frank.Hogrebe@hfpv-hessen.de 


\title{
Requirements engineering for the implementation of the OZG: status quo, case study and optimization potential for the implementation of locally relevant OZG solutions
}

\begin{abstract}
The act to improve online access to administrative services (called Onlinezugangsgesetz (OZG) in Germany) from 2017 requires, that public administrations must make their administrative services electronically accessible by the end of 2022. For implementation of this legal requirement, addressee-specific requirements form the essential solution-relevant basis. Federal government, federal states and municipalities have taken different routes to implement the OZG, whereby federal states and their municipalities play a key role in Germany due to the scope of administrative services. The article works out which framework applies to federal states when implementing the OZG and to what extent addressee-specific requirements are to be taken into account. Based on comparison results, a large municipal IT service provider is used to show which optimization potential can be exploited, provided that addressee-specific requirements are included in the implementation concept. Finally, recommendations for action are shown as to how chosen implementation paths in federal states and their municipalities could be optimized. The article addresses both, leaders and OZG experts in administrative practice as well as scientific community in this highly dynamic field of research.
\end{abstract}

Keywords Requirements engineering · Case study · Public administration · Online access act $\cdot$ Implementation routes

\section{Adressaten und Anforderungen des Onlinezugangsgesetzes}

Das Gesetz zur Verbesserung des Onlinezugangs zu Verwaltungsleistungen (kurz: Onlinezugangsgesetz - OZG) aus dem Jahr 2017 (Bundesministerium für Justiz und Verbraucherschutz. 2017) fordert, dass öffentliche Verwaltungen ihre Verwaltungsleistungen bis Ende 2022 auch elektronisch zugänglich machen. Ziel ist eine „flächendeckende Digitalisierung der Verwaltung Deutschlands bis 2022. Bis 2022 sollen Bund, Länder und die Kommunen alle Verwaltungsleistungen in Deutschland über Verwaltungsportale auch digital anbieten und diese Portale zu einem Verbund verknüpfen“ (Föderale IT-Kooperation 2021). Damit verpflichtet das OZG öffentliche Verwaltungen insgesamt in der jeweiligen Zuständigkeit ihre Verwaltungsleistungen bis Ende 2022 elektronisch zugänglich machen.

Dadurch kommen öffentliche Verwaltungen in die Rolle von potenziellen Auftraggebern von OZG-Softwarelösungen, falls und soweit konkrete Verwaltungsleistungen in eigener Zuständigkeit noch nicht elektronisch zugänglich sein sollten. Da das OZG Bund, Länder und Kommunen gleichermaßen adressiert, stellt sich die Frage, wie es erreicht werden kann, dass OZG-Softwareentwicklungen so ausgestaltet werden, dass diese adressatengerichtet eingesetzt werden können. Zur Konkretisierung der Frage, welche Verwaltungsleistungen konkret elektronisch zugänglich gemacht werden sollen, wurde im April 2018 ein OZG-Umsetzungskatalog veröffentlicht (Stocksmeier und Hunnius 2018), der zwischenzeitlich in eine interaktive 
Tab. 1 OZG Leistung „Führerschein“ mit ausgewählten Leika-Leistungen

OZG-Leistung „Führerschein“ (OZG-ID: 10169)

\begin{tabular}{|c|c|c|c|}
\hline Leika-Leistungen (Bezeichnung) & Leika-ID & $\begin{array}{l}\text { Leika- } \\
\text { Typ }^{\text {a }}\end{array}$ & $\begin{array}{l}\text { Regelungskompetenz (RV)/ } \\
\text { Vollzugskompetenz (VK) }\end{array}$ \\
\hline $\begin{array}{l}\text { Gnadenerweis bei Verkehrsordnungs- } \\
\text { widrigkeiten Erteilung }\end{array}$ & 99023004001000 & 4 & $\begin{array}{l}\mathrm{RV}=\text { Land } \\
\mathrm{VK}=\text { Land oder Kommune }\end{array}$ \\
\hline $\begin{array}{l}\text { Örtliches Fahrerlaubnisregister Aus- } \\
\text { kunft }\end{array}$ & 99024001023000 & $2 / 3$ & $\begin{array}{l}\mathrm{RV}=\text { Bund } \\
\mathrm{VK}=\text { Land oder Kommune }\end{array}$ \\
\hline $\begin{array}{l}\text { Eigenauskunft aus Zentralem Fahrer- } \\
\text { laubnisregister Erteilung }\end{array}$ & 99024003001000 & 1 & $\begin{array}{l}\mathrm{RV}=\text { Bund } \\
\mathrm{VK}=\text { Bund }\end{array}$ \\
\hline
\end{tabular}

Mehr als 70 weitere Leika-Leistungen

${ }^{\text {a} E i n e ~ t a b e l l a r i s c h e ~ A u f s t e l l u n g ~ u ̈ b e r ~ a l l e ~ L e i k a-T y p e n ~ f i n d e t ~ s i c h ~ b e i: ~ S t o c k s m e i e r ~ u n d ~ H u n n i u s ~(2018, ~}$ S. 8)

OZG-Informationsplattform überführt wurde (Bundesministerium des Innern und für Bau und Heimat 2021a), über die der OZG-Umsetzungskatalog kontinuierlich fortgeschrieben wird. Anpassungen an diesem Katalog obliegen dem IT-Planungsrat als zuständige Institution.

Als wesentliche Differenzierungsebene beinhaltet der OZG-Katalog sogenannte OZG-Leistungen. Der Beitrag behandelt die übergeordnete Fragestellung, ob die Ebene der OZG-Leistungen, als Bündelebene von bundes-, landes- und/oder kommunalrelevanten OZG-Einzelleistungen (sog. Leika-Leistungen), eine geeignete Ebene für die OZG-Softwareentwicklung für Kommunen bildet und welche Folgen sich daraus ergeben können, falls solche Softwareentwicklungen OZG-Lösungen umfassen (sollten), die Staatsebenen übergreifend sind. Aktuell umfasst dieser Katalog 573 OZG-Leistungen mit 7579 zugeordneten Leika-Leistungen ${ }^{1}$. So umfasst beispielsweise die OZG-Leistung „Führerschein“ (OZG-ID: 10169) mehr als 70 LeikaLeistungen, die sich in ihrer Zuständigkeit auf Bund, Länder und Kommunen verteilen. Tab. 1 illustriert dies beispielhaft ${ }^{2}$ :

Auch wenn postuliert wird, dass OZG-Leistungen insgesamt technisch realisierbar sind (Bundesministerium des Innern und für Bau und Heimat 2021b), so ist die Ebene der OZG-Leistungen für eine technische Umsetzung aus kommunaler Sicht zu hinterfragen. Denn OZG-Leistungen bündeln nicht selten bundes-, landes- und kommunalrelevante OZG-Einzelleistungen in einer Leistung, wie oben illustriert. Dieser Umstand kann die Adressaten gerichtete Identifikation von OZG-Lösungen erschweren, falls sich bei einer konkreten OZG-Leistung der Kreis der Adressaten Staatsebenen übergreifend darstellt. Mit der Folge, dass die Gewinnung von unterstützenden Experten aus der Verwaltungspraxis bei der Konzeption solcher OZGLösungen aufwendig wird, falls je nach OZG-Leistungsbündel unterschiedliche Experten aus verschiedenen Behörden benötigt werden. Auch werden Verwaltungen eher schwerer geneigt sein, OZG-Lösungen (kostenpflichtig) zu nutzen, falls in einer OZG-Lösung auch technische Bestandteile realisiert wurden, die nicht für die

\footnotetext{
1 Die konkreten Zahlen zu den OZG-Leistungen und Leika-Leistungen unterliegen einer Dynamik. Für die Zielsetzung und Aussagekraft dieses Beitrages ist dies aber nachrangig.

${ }^{2}$ Quelle: https://ozg.kdn.de/ozg-leistungen (dort findet sich auch eine Vielzahl weiterer Beispiele).
} 
Tab. 2 Potenzielle Kommunalrelevanz von Leika-Leistungen im OZG-Kontext

\begin{tabular}{lllll}
\hline Leika-Typ $^{\mathrm{a}}$ & Regelungskompetenz & Vollzugskompetenz & Summenwerte & $\begin{array}{l}\text { Potenziell kommunalrele- } \\
\text { vant }\end{array}$ \\
\hline 1 & Bund & Bund & 2627 & - \\
$2 / 3^{\mathrm{b}}$ & Bund & Länder/Kommunen & 4097 & \\
4 & Länder & Länder/Kommunen & 804 & 4952 \\
5 & Kommunen & Kommunen & 51 & \\
& & Summe gesamt & 7579 & \\
\hline
\end{tabular}

${ }^{a}$ Ein Leika-Typ 1 bedeutet, dass hier der Bund sowohl die Regelungs- als auch die Vollzugskompetenz hat. Ein Leika-Typ 5 bedeutet, dass hier die Kommunen die Regelungskompetenz- als auch die Vollzugskompetenz haben

${ }^{b}$ Hier sind 21 Leika-Typ-Leistungen enthalten, die unscharf einem Typ „1, 2/3“ auf der OZG-Informationsplattform zugeordnet wurden. Da damit auch Kommunen für die Vollzugskompetenz verantwortlich sein könnten, wurden diese hier mit summiert

eigene Zuständigkeit relevant sind, gleichwohl aber mit finanziert werden sollen. Dies kann damit auch die nachhaltige Entwicklung technischer Lösungen aus der Sicht von Dienstleistern erschweren.

Daher dienen OZG-Leistungen als erster Anhaltspunkt, geben aber noch nicht Auskunft darüber, wer wirklich für welche OZG-Leistung anteilig zuständig ist. Die Orientierung an Leika-Leistungen bietet hingegen ein Unterscheidungsmerkmal, wer für die elektronische Zugänglichmachung von Verwaltungsleistungen jeweils konkret zuständig ist (Bund, Länder oder Kommunen). Jedoch ist dies auf der Ebene der OZG-Informationsplattform nicht ausreichend differenziert. Tab. 2 summiert die Leika-Leistungen im OZG-Kontext, die potenziell kommunalrelevant sein könnten, im Überblick.

Deutlich wird, dass die fehlende Differenzierung in der Vollzugskompetenz zwischen Ländern und Kommunen es erforderlich macht, auf der Ebene der Bundesländer und der Kommunen zu klären, inwieweit die Länder von ihrer Vollzugskompetenz Gebrauch gemacht haben. Erst auf dieser Grundlage ist klar erkennbar, wer bezogen auf die 4952 potenziell kommunalrelevanten Leika-Leistungen im OZGKontext für die elektronische Zugänglichmachung von Verwaltungsleistungen jeweils konkret zuständig ist: Länder oder Kommunen.

Für das Requirements Engineering, die Anforderungsanalyse und -erhebung im Rahmen der Entwicklung erforderlicher OZG-Lösungen, sind dies zentrale Informationen. Requirements Engineering wird hier als umfassender Begriff zur Anforderungserhebung verstanden (Tiemeyer 2020, S. 358). Anforderungen sind grundlegend für die Beantwortung der Frage: Welche Eigenschaften ein zu entwickelndes Softwaresystem besitzen soll. „Sie beschreiben also das Problem, das gelöst werden soll“" (Balzert 2009, S. 437) oder wie es Partsch (1991, S. 25) formuliert: die Präzisierung der Problemstellung. Es geht daher um die Frage, welche Leika-Leistungen eine OZG-Softwarelösung technisch abdecken sollte. Dabei wird hier der Fokus auf die Ebene der Kommunen gelegt. Die Erhebung, welche Verwaltungsleistungen (auch Bündel dieser) durch eine OZG-Lösung technisch abgedeckt werden müssen, liefert auch wesentliche Informationen für Verwaltungen zur Beschaffung geeigneter OZG-Lösungen. Die Anforderungen spiegeln wesentliche Bedürfnisse, die bei der Entwicklung von Softwarelösungen berücksichtigt werden sollten. Glei- 
ches gilt für Softwarelösungen, die sich bereits in den Ländern und/oder Kommunen im technischen Einsatz befinden und zur Erfüllung des OZG geeignet sind.

Der Beitrag ist wie folgt aufgebaut. Nach den Ausführungen zu Adressaten und Anforderungen des OZG im Kapitel 1 befasst sich das nächste Kapitel 2 mit dem Status quo der OZG-Umsetzung in den Ländern. Im Kapitel 3 werden in der Folge anhand einer Fallstudie verschiedene Anforderungseffekte herausgearbeitet und diskutiert. Den Abschluss bildet das Kapitel 4 mit Handlungsempfehlungen.

\section{Status quo der OZG-Umsetzung in den Ländern}

Im Rahmen einer Erhebung mittels Onlinerecherche auf den offiziellen Internetseiten der Bundesländer wurde eruiert, wie sich die jeweiligen Bundesländer bis dato mit der Herausforderung befasst haben, die 4952 potenziell kommunalrelevanten Leika-Leistungen für ihr Bundesland hinsichtlich ihrer Vollzugskompetenz zwischen Land und Kommune herauszuarbeiten. Dass je nach Bundesland auf der Ebene der Kommunen noch eine weitergehende Differenzierung erforderlich ist, wird zu einem späteren Zeitpunkt in diesem Beitrag noch aufgegriffen (vgl. Abschn. 3.1). Insgesamt muss festgestellt werden, dass bis auf Nordrhein-Westfalen (NRW, ozg.kdn.de) in keinem Bundesland eine annähernd kommunalbezogene Differenzierung bis auf die Leika-Leistungsebene unmittelbar aus der Recherchearbeit festgestellt werden konnte (vgl. dazu auch: (Will 2020) Länder übergreifend, (Engel 2021) zu NRW, (Stockmann 2020) zu Rheinland-Pfalz, (Bickert et al. 2021) zu Baden-Württemberg oder (Synold 2020) zu Thüringen).

Grundsätzlich werden in der Außensicht der Bundesländer gleichsam OZG-Lösungen offeriert, die unter dem Rubrum „OZG“ über Serviceportale gebündelt werden (z.B. Bayern 2021a; Staatskanzlei Hamburg 2021). Ob bei den umfassenden OZG-Umsetzungsprojekten in den Bundesländern der Aspekt der Vollzugskompetenz unter Zugrundelegung des OZG-Umsetzungskataloges (vgl. Abschn. 1) berücksichtigt wurde, muss an dieser Stelle offenbleiben. Sollte dies nicht berücksichtigt worden sein, stellt sich die Frage, wie eine technische Umsetzung des Onlingzugangsgesetzes, ohne die initiale Kenntnis und Adressaten gerichtete Kommunikation der Zuständigkeit der 4952 potenziell kommunalrelevanten Leika-Leistungen zwischen Land und Kommunen, realisiert wird ${ }^{3}$.

Der OZG-Umsetzungskatalog (Bundesministerium des Innern und für Bau und Heimat 2021a) hat durch seine Orientierung an Leika-Leistungen ein Unterscheidungsmerkmal gewählt, wer für die elektronische Zugänglichmachung von Verwaltungsleistungen jeweils konkret zuständig ist (Bund, Länder oder Kommunen). Vor dem Hintergrund der kommunalen Selbstverwaltung gemäß Art. 28 Abs. 2 GG sind Kommunen in der Wahl der Umsetzung technischer Lösungen zunächst einmal frei. „Die Entscheidung über das „Ob“ besitzt die Gemeinde bei den Pflichtaufgaben nicht mehr, allein über das ,Wie“ der Aufgabenwahrnehmung besteht noch Entscheidungsfreiraum“ (Schäfer o.J.). Die Vollzugskompetenzen sind historisch gewachsen

\footnotetext{
3 Auch von Lucke (2021) stellt heraus, dass die konkreten Leika-Leistungen, anstatt der bündelnden OZGLeistungen, für die Verwaltungskunden relevanter sind.
} 
und deutlich vor der Verabschiedung des Onlinezugangsgesetzes festgelegt worden. Ungeachtet der Frage, ob mittels der Regelungskompetenzen von Bund und Ländern Vollzugskompetenzen neu geregelt werden können, ist gleichsam nicht davon auszugehen, dass solche Überlegungen bis Ende 2022, dem Stichtag zur Umsetzung des OZG, auch nur ansatzweise wahrscheinlich sind, geschweige denn technisch realisiert. Auch würden Regelungen hinsichtlich der Vollzugskompetenzen neben technisch-organisatorischen Fragen besonders auch rechtliche Fragen aufwerfen, deren Diskussion für die Zielsetzungen dieses Beitrages zu weit führen würden. So sind mit den von den Bundesländern auf die Kommunen übertragenen Vollzugskompetenzen auch Finanzzuweisungen verbunden, mit denen u.a. auch Personal in den Kommunen finanziert wird. Alleine in diesem Themenkomplex stellen sich diverse rechtliche Fragen, die bei einer Neuregelung von Vollzugskompetenzen zu beurteilen und rechtsverbindlich zu regeln wären ${ }^{4}$.

Daraus folgt, dass die Kommunen - bis auf weiteres - bei der Wahl ihrer Mittel zur technischen Umsetzung der ihnen über die Vollzugskompetenz zugewiesenen OZGVerwaltungsleistungen frei sind. Weiter ist zu berücksichtigen, dass es nicht „die Kommune" als solche gibt, sondern - je nach Bundesland - eine mehr oder weniger starke Differenzierung innerhalb der „Kommunalen Familie“ (dazu z. B. Volkmann 2019; oder Ünalgan 2020). So differenziert beispielsweise die Gemeindeordnung in NRW (Ministerium des Innern des Landes Nordrhein-Westfalen 2021) zwischen Kreisfreien Städten, Kreisen und kreisangehörigen Städten und Gemeinden, aber auch zudem zwischen Großen kreisangehörigen Städte und Mittleren kreisangehörigen Städten. Dem gegenüber differenziert die kommunale Gliederung im Bundesland Bayern zwischen Gemeinden, Kreisen und Bezirken aber zudem auch zwischen Großen Kreisstädten und kreisfreien Gemeinden ${ }^{5}$ (Bayern 2021b). Inwieweit OZGSoftwarelösungen für eine konkrete Kommune relevant sind und damit von Interesse sein sollten, hängt folglich davon $a b$, welche Verwaltungsleistungen (mit OZGBezug sind dies Leika-Leistungen bzw. Bündel dieser) die jeweilige Lösung konkret abdeckt und ob dafür gegebenenfalls bereits ganz oder teilweise vorhandene Lösungen in der konkreten Kommune vorhanden sind.

Da die Kommunen als Adressaten von OZG-Lösungsangeboten letztlich entscheiden, ob sie eine Lösung einsetzen werden oder nicht, wäre eine Lösungsentwicklung ohne konkrete Kenntnis darüber, ob das technisch angestrebte Leistungsbündel mit dem Bedarf vor Ort überhaupt korreliert, zumindest als nicht risikolos zu bewerten. Damit aber hier kein Missverständnis entsteht: es ist natürlich kaum möglich alle örtlichen Spezifika bei einer Softwareentwicklung zu berücksichtigen, gleichsam sollte für eine Kommune schon klar erkennbar sein, welche Leika-Leistungen mit welcher OZG-Lösung zugänglich gemacht werden.

Auf dieser Basis ist es Kommunen möglich, zu entscheiden, ob ein Lösungsangebot aus individueller, kommunaler Sicht einen Mehrwert darstellt (oder nicht), stets unter Einbeziehung der zum Zeitpunkt der Entscheidung bereits vorhande-

\footnotetext{
${ }^{4}$ Die Regelungskompetenz leitet sich aus der Gesetzgebungskompetenz ab, die Vollzugskompetenz aus der operativen Umsetzung gesetzlicher Regelungen, vgl. im Weiteren dazu z. B. (Winter o.J.).

5 Auf den Aspekt der sog. Verwaltungsgemeinschaften, als Zusammenschluss von kleineren Gemeinden, sei hingewiesen, diese bilden jedoch keinen eigenen Gemeindetyp (Bayern 2021b).
} 
nen OZG-konformen Lösungen vor Ort. Sofern Kommunen in Deutschland bereits Formularanbieter beauftragt haben, die - je nach Anbieter am Markt - bereits eine Vielzahl OZG-konformer Formulare(-assistenten) anbieten können, ist die Frage des Mehrwertes aus kommunaler Sicht um so relevanter, denn über solche OZG-konformen Formularangebote bereits elektronisch zugänglich gemachten Leika-Leistungen erfordern eben nicht zwingend einen Umstieg auf eine andere, gegebenenfalls neu entwickelte und angebotene OZG-Lösung. Ein Aspekt, der besonders bei der Entwicklung von (anschub)finanzierten OZG-Lösungen durch Bund und/oder Länder nicht unberücksichtigt bleiben sollte (vgl. Bundesministerium des Innern und für Bau und Heimat 2020).

\section{Fallstudie und Anforderungseffekte}

\subsection{Studiendesign}

Im Rahmen einer wissenschaftlichen Begleitforschung bei einem kommunalen ITDienstleister in NRW, dessen Verbandsgebiet 40 Kommunen unterschiedlicher Größenordnung umfasst, wurde im Jahr 2019 ein umfassendes Projekt zur Umsetzung des Onlinezugangsgesetzes gestartet. Dies mit der Zielsetzung, bis Ende 2022 die gesetzlichen Anforderungen im Verbandsgebiet umzusetzen. Das Projekt startete im Frühjahr 2019 mit 5039 Leika-Leistungen (Stand: 06.03.2019), die bis zum 27.03.2021 auf 7549 Leika-Leistungen angewachsen sind. In einer klassischen Projektorganisation mit Arbeitsgruppen, Zeit- und Aktivitätenplanung wurden auf Basis der jeweils aktuellen Excel-Exporte aus der OZG-Informationsplattform (Bundesministerium des Innern und für Bau und Heimat 2021a; vgl. Abschn. 1) die Datensammlungen mit folgenden Fragestellungen untersucht:

1. Welche der 7549 Leika-Leistungen sind kommunalrelevant? Wo liegt die Vollzugskompetenz für die Umsetzung von Leika-Leistungen allgemein bei den Kommunen (d.h. nicht bei Land od. Bund)?

2. Wie verteilen sich die Leika-Leistungen auf (a) bürgerbezogene (G2C), (b) unternehmensbezogene (G2B) Verwaltungsleistungen sowie (c) G2B/C-Verwaltungsleistungen, also auf jene, die sowohl für Bürger*innen als auch für Unternehmen relevant sein können? Die Abkürzungen stehen für „Government-to-Business - G2B“ und „Government-to-Citizen - G2C“ (von Lucke und Reinermann 2000, S. 2).

3. Wie verteilen sich die Leika-Leistungen, wenn diese in (i) „Formularleistungen“, (ii) „Meldeleistungen“ und (iii) „Bündelleistungen“ kategorisiert werden? Dies vor dem Hintergrund, dass, Leika-Leistungen nach (iii) bereits dadurch erfüllt werden, dass die einem Bündel zugeordneten Leika-Leistungen (enden auf „0000“) aus (i) und (ii) elektronisch zugänglich sind.

4. Für welchen Kommunaltyp sind die kommunalbezogenen Leika-Leistungen relevant? Welches Bündel an Leika-Leistungen liegt in der konkreten Zuständigkeit einer Kommune und/oder einer bestimmten kommunalen Größenordnung? 
Den Fragestellungen ging eine umfassende Analyse der einschlägigen Literaturund Praxisquellen voraus, die im Dialog mit den kommunalen Vertretern sukzessive erörtert und konkretisiert wurden. Dabei wurden auf der Ebene der OZG-Themenfelder Arbeitsgruppen gebildet und die Fragestellungen über einen Zeitraum von 18 Monaten mittels strukturierter Workshops bearbeitet. Im Folgenden sollen Aspekte besonders hervorgehoben werden, welche die Gründe für die Auswahl der Fragestellungen ( 1 bis 4$)$ verdeutlichen:

- Fragestellung 1 diente dazu, zunächst die Leika-Leistungen zu extrahieren, die überhaupt in der Zuständigkeit der kommunalen Sphäre liegen, denn nur diese sind von Kommunen bis Ende 2022 elektronisch zugängig zu machen. Dieser Schritt konnte kommunalübergreifend bearbeitet werden.

- Fragestellung 2 trug dem Umstand Rechnung, dass in den Arbeitsgruppen unternehmensbezogene Verwaltungsleistungen nicht ausreichend beurteilt werden konnten. Da die Teams zu Beginn primär mit Experten aus den Bereichen Digitalisierung, IT und Organisation besetzt waren, fehlte oftmals der spezifische Sachverstand der kommunalen Wirtschaftsförderungen. Um einschätzen zu können, welchen Anteil die G2B- und G2B/C-Verwaltungsleistungen im Verhältnis zu den Bürger*innen bezogenen (G2C) Verwaltungsleistungen haben, wurde die Aufteilung zur Fragestellung 2 vorgenommen. Dies mit der Zielsetzung, die kommunalen Wirtschaftsförderungseinheiten begleitend in die Umsetzung des OZG einbeziehen zu können, da dort die Unternehmensrelevanz des OZG spezifiziert werden kann.

- Fragestellung 3 bildete den Einstieg in die technische Umsetzung des OZG. Unter „Meldung(leistungen)“ wurden solche Verwaltungsleistungen subsumiert, die üblicherweise nicht (über Formulare) beantragt werden, jene hingegen wurden unter „Formular(leistungen)“ erfasst. „Bündel(leistungen)“ bilden eine Leika-Leistungsebene zur Zusammenfassung von Melde- und/oder Formularleistungen. Vor dem Hintergrund, dass in diesem konkreten Verbandsgebiet alle Kommunen einen Rahmenvertrag mit einem Formularanbieter hatten, der eine Vielzahl von OZGkonformen Formularen bereits bereitstellen kann, bot es sich an, systematisch zu erfassen, welche der kommunalrelevanten Leika-Leistungen bereits durch (bloßes) „Freischalten“ der OZG-konformen Onlineformulare auf der kommunalen Homepage zugänglich gemacht werden können. Konkret ermöglicht die Kategorisierung zu (3) eine Zuordnung von Leika-Leistungen zu den über den Anbieter nutzbaren Formularassistenten.

- Fragestellung 4 war die Antwort auf den in den Arbeitsgruppen herausgestellten individuellen Blick auf das OZG, mit dem Fokus, welche Leika-Leistungen denn von einer konkreten Kommune zugänglich zu machen sind. Hier sind die kommunalen Aufgabenverteilungen in den Bundesländern nicht identisch. In NRW differenziert die Gemeindeordnung (Ministerium des Innern des Landes Nordrhein-Westfalen 2021) zwischen Kreisfreien Städten, Kreisen und kreisangehörigen Städten und Gemeinden, aber auch zudem zwischen Großen kreisangehörigen Städte und Mittleren kreisangehörigen Städten, die in ihrem Gebiet aufgrund der eigenen Leistungsfähigkeit einen Bereich von ansonsten auf der Kreisebene angesiedelte Aufgaben eigenverantwortlich wahrnehmen (z.B. als Untere Bauaufsichtsbehörde oder als eigenes Jugendamt). 
Tab. 3 Kommunalrelevante Leika-Leistungen differenziert nach Zielgruppen und Zugangsarten

\begin{tabular}{|c|c|c|c|c|c|c|c|}
\hline $\begin{array}{l}\text { Leika- } \\
\text { Leistungen } \\
\text { kommunal } \\
\text { (potenziell) }\end{array}$ & $\begin{array}{l}\text { (1) Leika- } \\
\text { Leistungen } \\
\text { kommunal } \\
\text { (Fallstudie) }\end{array}$ & \multicolumn{3}{|c|}{$\begin{array}{l}\text { (2) Leika-Leistungen differenziert } \\
\text { nach Zielgruppen der Verwal- } \\
\text { tungsleistungen }\end{array}$} & \multicolumn{3}{|c|}{$\begin{array}{l}\text { (3) Leika-Leistungen diffe- } \\
\text { renziert nach Zugangsart der } \\
\text { Verwaltungsleistung }\end{array}$} \\
\hline \multirow[t]{3}{*}{4952} & 2799 & G2B & $\mathrm{G} 2 \mathrm{~B} / \mathrm{C}$ & G2C & Meldung & Formular & Bündel \\
\hline & & 594 & 727 & 1478 & 138 & 1948 & 713 \\
\hline & & \multicolumn{3}{|c|}{2799} & \multicolumn{3}{|c|}{2799} \\
\hline
\end{tabular}

Diese für NRW bedeutsame Kommunalstruktur und Aufgabenverteilung im kreisangehörigen Raum, ist Ergebnis der nach der Gebietsreform in den 70er-Jahren des letzten Jahrhunderts in den 80er-Jahren durchgeführten Kommunalen Funktionalreform. Basis des NRW-Zuständigkeitssystems im kreisangehörigen Bereich ist der $§ 4$ GO NRW im Zusammenhang mit den darauf basierenden spezialgesetzlich erfolgten Aufgabenzuweisungen, der seinerzeit in den 3 Funktionalreformgesetzen durch den Landtag eingefügt wurde. Bei der Bildung der OZG-Themenfeld bezogenen Arbeitsgruppen wurde bei der Zusammensetzung darauf geachtet, dass entsprechend der kommunalen Aufgabenverteilungen jeweils entsprechende Expert*innen in den Gruppen vertreten waren.

\subsection{Kernergebnisse}

Tab. 3 zeigt die kommunalrelevanten Leika-Leistungen differenziert nach den Fragestellungen (1-3) im Überblick. In der Fallstudie waren bis auf „Kreisfreie Städte“ alle Differenzierungen vertreten, also vier der fünf „Kommunaltypen“ von NRWKommunen. Die Ergebnisse zur Fragestellung 4 folglich in Tab. 4.

Aus Tab. 3 lassen sich verschiedene zentrale Erkenntnisse ableiten:

1. Von den 4952 potenziell kommunalrelevanten Leika-Leistungen sind mit 56,52\% (aus: 2799/4952×100) mehr als die Hälfte in der Vollzugskompetenz der Kommunen.

Tab. 4 Kommunalrelevante OZG-Einzelleistungen differenziert nach Kommunalen Größenklassen

\begin{tabular}{|c|c|c|c|c|c|}
\hline $\begin{array}{l}\text { Leika-Leistun- } \\
\text { gen kommunal }\end{array}$ & $\begin{array}{l}\text { Leika-Leistun- } \\
\text { gen kommunal }\end{array}$ & $\begin{array}{l}\text { (4) Le } \\
\text { klasse }\end{array}$ & $\begin{array}{l}\text { istungen d } \\
\text { em. GO N }\end{array}$ & ziert nach Kor & unalen Größen- \\
\hline 4952 & 2799 & Kreis & $\begin{array}{l}\text { Große } \\
\text { kreisan- } \\
\text { gehörige } \\
\text { Stadt }\end{array}$ & $\begin{array}{l}\text { Mittlere } \\
\text { kreisangehö- } \\
\text { rige Stadt }\end{array}$ & $\begin{array}{l}\text { Kreisangehörige } \\
\text { Stadt oder Ge- } \\
\text { meinde i. W. }\end{array}$ \\
\hline & & 2190 & 1543 & 1157 & 1031 \\
\hline
\end{tabular}

Hinweis: Da Verwaltungsleistungen nicht ausschließlich einer Größenklasse zugeordnet sind, sondern für eine Verwaltungsleistung auch Kommunen mehrere Größenklassen zuständig sind, sind die Zahlenwerte in Tab. 4 nicht aufzusummieren und auch nicht untereinander in Bezug zu setzen. Gleichsam kann der Anteil einer Spalte an den übergreifenden Bezugswert 2799 (Leika-Leistungen kommunal-Fallstudie) hergestellt werden.

${ }^{a}$ Kreisfreie Städte sind in dieser Tabelle nicht enthalten, da diese nicht Teil der Fallstudie waren 
2. Von diesen 2799 kommunalrelevanten Leika-Leistungen sind 1478 Bürger*innen bezogene (G2C-) Verwaltungsleistungen und 1321 (aus: 594+727) unternehmensbezogene (G2B- und G2B/C-) Verwaltungsleistungen.

3. Rund 25,5\% (aus: 713/2799×100) der kommunalrelevanten Leika-Leistungen sind Bündelleistungen. Mit 1948 Leika-Leistungen der Zugangsart „Formular(Leistung)“" sind mit Abstand die meisten Leika-Leistungen (bereits) dadurch elektronisch umsetzbar, indem OZG-konforme Formular(assistenten) den Bürger*innen und Unternehmen zur Verfügung gestellt werden.

4. Tab. 4 zeigt die kommunalrelevanten Leika-Leistungen differenziert nach der Gemeindeordnung NRW (Ministerium des Innern des Landes Nordrhein-Westfalen 2021) im Überblick und unter Bezug auf die Fragestellung (4):

Hinsichtlich des Engagements einzelner Kommunen ist die konkrete Relevanz von Bedeutung: Tab. 4 gliedert dazu die kommunalrelevanten Leika-Leistungen auf nach der in NRW nach der Gemeindeordnung (Ministerium des Innern des Landes Nordrhein-Westfalen 2021) rechtlich geregelten Differenzierung zwischen Kreisen, Großen kreisangehörigen Städte, Mittleren kreisangehörigen Städten und kreisangehörigen Städten und Gemeinden im Übrigen. In der Fallstudie waren „Kreisfreie Städte“ nicht vertreten, so dass diese nicht einbezogen wurden. Entsprechende Differenzierungen können für andere Bundesländer vergleichbar abgeleitet werden.

Für die technische Umsetzung des OZG liefert die Fallstudie grundlegende Erkenntnisse für das Requirements Engineering:

- Einerseits ist als Ergebnis der Fallstudie nun nicht nur bekannt, welche LeikaLeistungen kommunalrelevant sind, sondern auch, welche von diesen (lediglich) mit OZG-konformen Formular(assistenten) umgesetzt werden können.

- Zum anderen wird damit auch klar, dass es hinsichtlich notwendiger OZG-Lösungen (nur) noch darum gehen könnte, welche Lücken sich im Umsetzungsportfolio einer Kommune zeigen, denn nur für diese wären Überlegungen anzustellen, wie diese technisch umgesetzt werden sollten, um den Bürger*innen und Unternehmen auch noch fehlende Verwaltungsleistungen elektronisch zugänglich zu machen.

- Weiter wird klar, dass die Menge der kommunalrelevanten Leika-Leistungen umfangreich ist. Hier kann es von Vorteil sein, fehlende Verwaltungsleistungen in einer größeren Gemeinschaft anzugehen.

Für das Requirements Engineering ergibt sich hieraus zudem eine weitere Information, denn für die Umsetzung technischer Anforderungen, ist es zwingend, dass ein Auftraggeber vorhanden ist, der die mit einer Beschaffung beziehungsweise Entwicklung verbundenen Kosten trägt. Neben Aspekten der Anschubfinanzierung aus Landes- und/oder Bundesmitteln ist es damit die Nachhaltigkeit von Finanzierung, die hier mit hineinspielt.

Die an der Fallstudie beteiligten 40 Kommunen können für jede Leika-Leistung klar beurteilen, ob diese (überhaupt) in ihrem (Vollzugs)Zuständigkeitsbereich liegt und falls ja, ob diese nicht bereits durch vorhandene OZG-Lösungen (bspw. OZGkonforme Formulare) abgedeckt ist. In solchen Fällen wird es wohl schwer(er), 
Kommunen für die (kostenpflichtige) Nutzung von bisher örtlich nicht eingesetzten OZG-Lösungen zu gewinnen.

\section{Handlungsempfehlungen}

Der Beitrag arbeitet entlang von 4 Fragestellungen die Bedeutung von Leika-Leistungen im OZG-Kontext heraus und beleuchtet, inwieweit Adressaten spezifische Anforderungen relevant sind. Auf Basis von Vergleichsergebnissen eines großen kommunalen IT-Zweckverbandes werden Optimierungspotenziale diskutiert, sofern adressatenspezifische Anforderungen bereits bei der Umsetzungskonzeption einbezogen werden. Besonders die Kenntnis von kommunal-individuellen „OZG-Zuständigkeitsbündeln“" verschafft Kommunen wie Dienstleistern der OZG-Softwareentwicklung Klarheit darüber, wo anderenorts entwickelte beziehungsweise in der Entwicklung befindlichen OZG-Lösungen für eine gesetzeskonforme Umsetzung des OZG für Kommunen (überhaupt) relevant sind.

Folgende Handlungsempfehlungen können aus den Erkenntnissen in diesem Beitrag aufgezeigt werden:

- Für Kommunen und Kommunale IT-Dienstleister: Vor dem Hintergrund, dass OZG-Leistungen regelmäßig bundes-, landes- und/oder kommunalbezogene Leika-Leistungen im OZG-Kontext als Bündel umfassen können, sollte geprüft werden, welche Kommunalrelevanz eine OZG-Lösung am Markt tatsächlich hat. Dies auch vor dem Hintergrund, dass konkrete Verwaltungsleistungen auch mit örtlich bereits im Einsatz befindlichen Softwarelösungen OZG-konform sein können.

- Für Dachverbände Kommunaler IT-Dienstleister: Soweit in einem Bundesland derartige Dachverbände existieren (wie der KDN in NRW) und kommunalbezogene OZG-Lösungen entwickelt werden sollen, sollte die vorgenannte Kommunalrelevanz einer OZG-Lösung auf der Ebene der Leika-Leistungen entwickelt und beurteilt werden. Dies umso mehr, falls die Nachhaltigkeit der Finanzierung kommunalbezogener OZG-Lösungen eine relevante Determinante bildet.

- Für Bundesländer: Die gewählten OZG-Umsetzungskonzepte in den Bundesländern sollten daraufhin überprüft werden, inwieweit geplante OZG-Lösungen die bundeslandspezifische Aufgabenverteilung und festgelegte Vollzugskompetenz zwischen Land und Kommunen berücksichtigt. Dies umso mehr, falls (auch hier) die Nachhaltigkeit der Finanzierung kommunalbezogene OZG-Lösungen eine relevante Determinante bildet. Gleiches gilt für Softwareunternehmen, die an OZGLösungen beteiligt sind.

Für die OZG-Umsetzungskonzepte im Bund und in den Ländern bedeutet dies insgesamt, dass für die Ebene der Kommunen die kommunal-individuellen Zuständigkeitsfragen klar beantwortet sein sollten, will man vermeiden, dass anschubfinanzierte OZG-Lösungen gegebenenfalls nicht, nicht umfänglich oder nicht nachhaltig von den adressierten Kommunen angenommen, weitergenutzt und (letztlich dauerhaft) weiterfinanziert werden. Anders würde sich dies darstellen, falls (öffentliche) Auftraggeber in Bund und Land dauerhaft die Kosten für kommunale OZG-Lösun- 
gen tragen wollten, was auf Basis der bisherigen Erkenntnisse jedoch eher nicht zu erwarten ist.

Funding Open Access funding enabled and organized by Projekt DEAL.

Open Access Dieser Artikel wird unter der Creative Commons Namensnennung 4.0 International Lizenz veröffentlicht, welche die Nutzung, Vervielfältigung, Bearbeitung, Verbreitung und Wiedergabe in jeglichem Medium und Format erlaubt, sofern Sie den/die ursprünglichen Autor(en) und die Quelle ordnungsgemäß nennen, einen Link zur Creative Commons Lizenz beifügen und angeben, ob Änderungen vorgenommen wurden.

Die in diesem Artikel enthaltenen Bilder und sonstiges Drittmaterial unterliegen ebenfalls der genannten Creative Commons Lizenz, sofern sich aus der Abbildungslegende nichts anderes ergibt. Sofern das betreffende Material nicht unter der genannten Creative Commons Lizenz steht und die betreffende Handlung nicht nach gesetzlichen Vorschriften erlaubt ist, ist für die oben aufgeführten Weiterverwendungen des Materials die Einwilligung des jeweiligen Rechteinhabers einzuholen.

Weitere Details zur Lizenz entnehmen Sie bitte der Lizenzinformation auf http://creativecommons.org/ licenses/by/4.0/deed.de.

\section{Literatur}

Balzert (2009) Lehrbuch der Softwaretechnik: Basiskonzepte und Requirements Engineering. Spektrum Akademischer Verlag, Heidelberg

Bayern (2021a) Bayern Portal. https://www.freistaat.bayern/. Zugegriffen: 11. Juli 2021

Bayern (2021b) Kommunale Gliederung. https://www.stmi.bayern.de/kub/kommunalegliederung/index. php. Zugegriffen: 11. Juli 2021

Bickert, Brand, Erath, Grothe, Häge, Hagel, Herrmann, Kiselev, Kratzer, Leser, Schütz, Seiz, Siller, Treuer Waibel (2021) OZG-Leistungs-Analyseprojekt. Ein Beitrag zur OZG-Umsetzung in Baden-Württemberg, 3. Februar 2021. Fautz, Götz, Kozel, Laue, Leikowski, Maier, Müller-Török (Hrsg.). Hochschule für öffentliche Verwaltung und Finanzen Ludwigsburg, Ludwigsburg

Bundesministerium des Innern, für Bau und Heimat (Hrsg) (2021a) OZG-Informationsplattform. Resource dokument. https://informationsplattform.ozg-umsetzung.de/iNG/app/intro. Zugegriffen: 11. Juli 2021

Bundesministerium des Innern, für Bau und Heimat (Hrsg) (2021b) OZG-Informationsplattform. Resource dokument. https://leitfaden.ozg-umsetzung.de/display/OZG/2.1+Verwaltungsleistungen+im+Sinne+ des+OZG. Zugegriffen: 11. Juli 2021

Bundesministerium für Justiz und Verbraucherschutz. (Hrsg) (2017) Gesetz zur Verbesserung des Onlinezugangs zu Verwaltungsleistungen (Onlinezugangsgesetz - OZG). Resource dokument. https://www. gesetze-im-internet.de/ozg/BJNR313800017.html. Zugegriffen: 11. Juli 2021

Engel (2021) Die Umsetzung des Onlinezugangsgesetzes in Nordrhein-Westfalen aus kommunaler Perspektive. Handbuch Onlinezugangsgesetz, S 269-294

Föderale IT-Kooperation (2021) Flächendeckende Digitalisierung der Verwaltung Deutschlands bis 2022. Resource dokument. https://www.it-planungsrat.de/DE/ITPlanungsrat/OZG-Umsetzung/ OZG_Umsetzung_node.html. Zugegriffen: 11. Juli 2021

von Lucke, Reinermann (2000) Speyerer Definition von Electronic Government. Forschungsinstitut für öffentliche Verwaltung bei der Deutschen Hochschule für Verwaltungswissenschaften Speyer (Hrsg.). http://www.joernvonlucke.de/ruvii/Sp-EGov.pdf. Zugegriffen: 11. Juli 2021

von Lucke (2021) Die Wissenschaft Verwaltungsinformatik und das Onlinezugangsgesetz. Handbuch Onlinezugangsgesetz, S 119-144

Ministerium des Innern des Landes Nordrhein-Westfalen (2021) Gemeindeordnung für das Land Nordrhein-Westfalen (GO NRW). https://recht.nrw.de/lmi/owa/pl_text_anzeigen?v_id=23200212051034 38063. Zugegriffen: 11. Juli 2021

Partsch (1991) Requirements Engineering. Handbuch der Informatik, Bd. 5.5. Oldenbourg, Münden, Wies

Schäfer Kommunale Selbstverwaltung. Gabler Wirtschaftslexikon. Resource dokument. https://wirtschafts lexikon.gabler.de/definition/kommunale-selbstverwaltung-41347. Zugegriffen: 11. Juli 2021 
Staatskanzlei Hamburg (2021) Digitale Stadt. Städtische Digitalisierungsprojekte. https://www.hamburg. de/senatskanzlei/digitalstrategie-fuer-hamburg/13505076/staedtische-digitalisierungsprojekte/. Zugegriffen: 11. Juli 2021

Stockmann (2020) Die Umsetzung des Onlinezugangsgesetzes auf kommunaler Ebene: Untersuchung des Potentials der Digitalisierung anhand ausgewählter Kommunen in Rheinland-Pfalz. Speyerer Arbeitshefte Nr. 240. WITI-Berichte Nr. 3. Deutsche Universität für Verwaltungswissenschaften, Speyer

Stocksmeier, Hunnius (2018) OZG-Umsetzungskatalog. Digitale Verwaltungsleistungen im Sinne des Onlinezugangsgesetzes. ]init[ AG (Hrsg.) im Auftrag des Bundesministeriums des Innern, für Bau und Heimat. https://www.it-planungsrat.de/SharedDocs/Downloads/DE/Entscheidungen/26_Sitzung/ TOP2_Anlage_OZGUmsetzungskatalog.pdf?_blob=publicationFile\&v=4. Zugegriffen: 11. Juli 2021

Synold (2020) Implementation des Onlinezugangsgesetzes auf kommunaler Ebene im Freistaat Thüringen. Speyerer Arbeitshefte Nr. 247. WITI-Berichte Nr. 6. Deutsche Universität für Verwaltungswissenschaften, Speyer

Tiemeyer (2020) Handbuch IT-Management. Konzepte, Methoden, Lösungen und Arbeitshilfen für die Praxis. Hanser, München

Ünalgan (2020) Das Gesetz zur Verbesserung des Onlinezugangs zu Verwaltungsleistungen (OZG). Eine empirische Untersuchung am Beispiel der Städte Dortmund und Schwerte. Ruhr-Universität Bochum, Fakultät für Sozialwissenschaft, Bochum

Volkmann (2019) Anforderungen und Auswirkungen des Onlinezugangsgesetzes - eine Analyse am Beispiel von Limbach-Oberfrohna. Hochschule Meißen (FH), Meißen

Will (2020) Kommunales E-Government im kooperativen Föderalismus: Die vertikale Koordination zwischen Kommunal- und Landesebene hinsichtlich einer effizienten und einheitlichen Umsetzung des Onlinezugangsgesetzes. Institut für Politikwissenschaft. Technische Universität Darmstadt, Darmstadt

Winter Gesetzgebungskompetenz, Definition: Was ist „Gesetzgebungskompetenz“? Gabler Wirtschaftslexikon. Springer Fachmedien Wiesbaden. https://wirtschaftslexikon.gabler.de/definition/gesetzgebungs kompetenz-33145. Zugegriffen: 11. Juli 2021 